\title{
Testosterone use causing erythrocytosis
}

\author{
Andrea Cervi MD MSc, Amaris K. Balitsky MD MSc
}

Cite as: CMAJ 2017 October 16;189:E1286-8. doi: 10.1503/cmaj.170683

A 60-year-old man with a medical history of depression, seasonal allergies, remote appendectomy and cholecystectomy presented to the emergency department with a sudden headache. Upon examination, his blood pressure was elevated (190/112 mm Hg) and he had erythrocytosis (hemoglobin 196 [normal 130-180] g/L and hematocrit 58\% [normal 40\%54\%]). Results for a complete blood cell count that was obtained six months before the patient's visit to the emergency department were normal (hemoglobin $154 \mathrm{~g} / \mathrm{L}$ and hematocrit 46\%). Our patient was discharged from the emergency department with a prescription for amlodipine (5 mg once daily) for management of his hypertension. After follow-up with his family physician, he was referred to our general hematology clinic for evaluation of erythrocytosis.

We noticed that the patient was using a topical formulation of testosterone at two pumps (125 mg per pump) twice daily for fatigue and perceived androgen deficiency, although his pretreatment level of total testosterone was within normal range at 17 (normal 7.6-31.4) nmol/L. He was also taking citalopram and, on occasion, lorazepam. He reported intermittent facial flushing, but he had not experienced focal neurologic deficits to suggest stroke, tinnitus, vertigo, abdominal pain, cardiac symptoms or other symptoms consistent with hyperviscosity syndrome.

There was nothing on history or physical examination to suggest a primary hematologic cause for erythrocytosis. Upon inquiry, the patient did not have constitutional symptoms, erythromelalgias (e.g., pain, redness and elevated temperature of extremities), aquagenic pruritus (e.g., itching that occurs upon contact with water, without visible lesions), or early satiety or abdominal bloating suggestive of splenomegaly. In addition, his cardiac and respiratory examinations were unremarkable. He had no risk factors for chronic hypoxia, such as a smoking history, or lung or cardiac pathology.

Results for peripheral blood testing for the JAK2 mutation, which is present in about $97 \%$ of patients with polycythemia vera, ${ }^{1}$ were negative. Moreover, our patient's serum erythropoietin level was inappropriately normal at 9.6 (normal 3.8-16.9) IU/L, which further supported that a secondary process was causing elevated hemoglobin and hematocrit through an erythropoietin-dependent mechanism. Renal ultrasonography to exclude the presence of an erythropoietin-secreting tumour was unremarkable.

Because of the negative result for JAK2 testing, inappropriately normal serum erythropoietin level and temporal association with onset of androgen replacement therapy in our patient,

\section{KEY POINTS}

- Testosterone therapy can cause secondary erythrocytosis.

- Erythrocytosis can cause symptoms of hyperviscosity, such as headache, fatigue, blurred vision and paresthesias.

- A baseline value for hematocrit should be obtained before testosterone therapy is started, and serial values should be taken at 3, 6 and 12 months after initiation of treatment.

- For symptomatic patients with a hematocrit value over $54 \%$, testosterone should be discontinued and phlebotomy considered.

we diagnosed erythrocytosis secondary to testosterone supplementation, and we recommended that he discontinue treatment with testosterone. Because his hematocrit was $58 \%$ in the setting of new onset hypertension, we also suggested urgent phlebotomy, after which his hemoglobin level and hematocrit normalized (154 g/L and 45\%, respectively) (Figure 1).

\section{Discussion}

The Canadian Men's Health Foundation Multidisciplinary Guidelines Task Force on Testosterone Deficiency ${ }^{2}$ and the American Endocrine Society ${ }^{3}$ recommend testosterone for men with symptomatic androgen deficiency and low levels of serum testosterone. Erythrocytosis is a predictable yet underrecognized effect of testosterone. ${ }^{2,3}$

Erythrocytosis is an increase in the number of erythrocytes and is defined as a hemoglobin level above $185 \mathrm{~g} / \mathrm{L}$ and hematocrit percentage over $49 \%$ in men, or $165 \mathrm{~g} / \mathrm{L}$ and $48 \%$, respectively, in women. ${ }^{1}$ An approach to erythrocytosis includes distinguishing a primary bone marrow disorder (e.g., polycythemia vera or other myeloproliferative neoplasm) from possible secondary causes. Primary erythrocytosis is characterized by a low level of serum erythropoietin and secondary causes by normal or high erythropoietin (Figure 2).

Secondary erythrocytoses are further subdivided into congenital and acquired. Congenital causes include germline mutations resulting in high oxygen affinity hemoglobinopathies, altered intracellular oxygen sensing pathways or enhanced erythropoietin receptor signalling. More commonly, erythrocytosis is acquired. Erythropoietin stimulation can be physiologically appropriate in the setting of chronic hypoxic states such as lung disease, heavy smoking, intracardiac shunting, hypoventilation syndromes and local renal hypoxia (e.g., in renal artery stenosis). Drugs to stimu- 


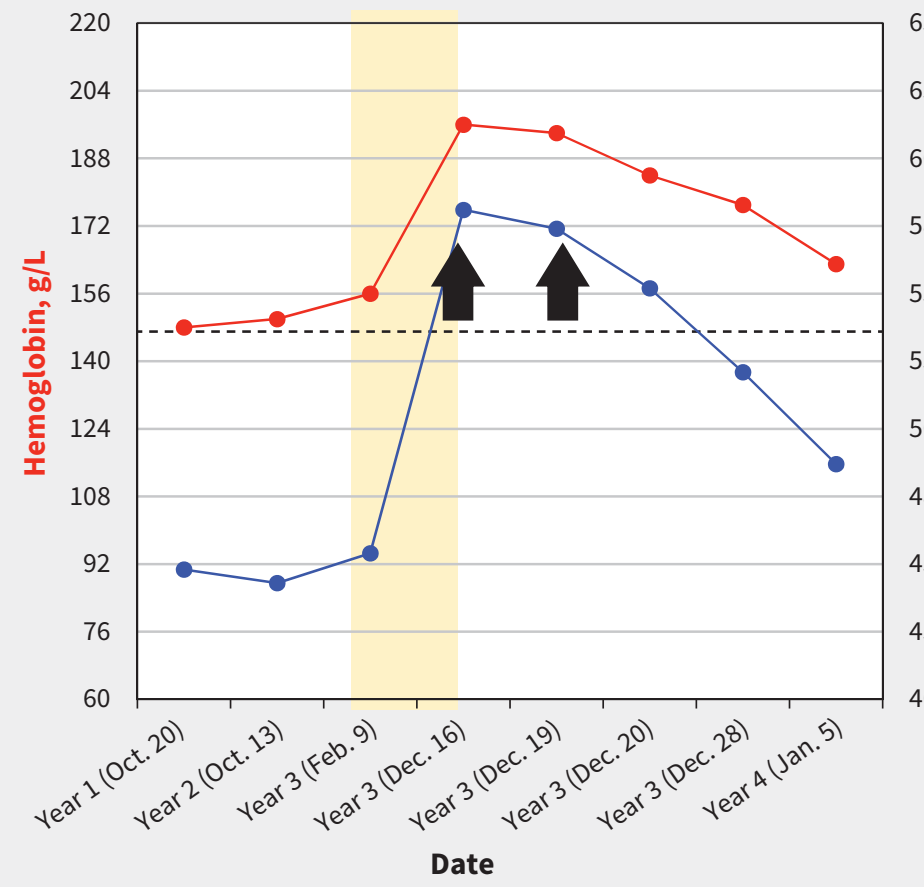

Figure 1: Trends for hemoglobin level (red line) and hematocrit percentage (blue line) in a 65-year-old man receiving testosterone supplementation for perceived androgen deficiency. Shaded section indicates duration of time that the patient was taking testosterone, and black arrows indicate when phlebotomy was conducted. Broken black line indicates a hematocrit of $54 \%$.

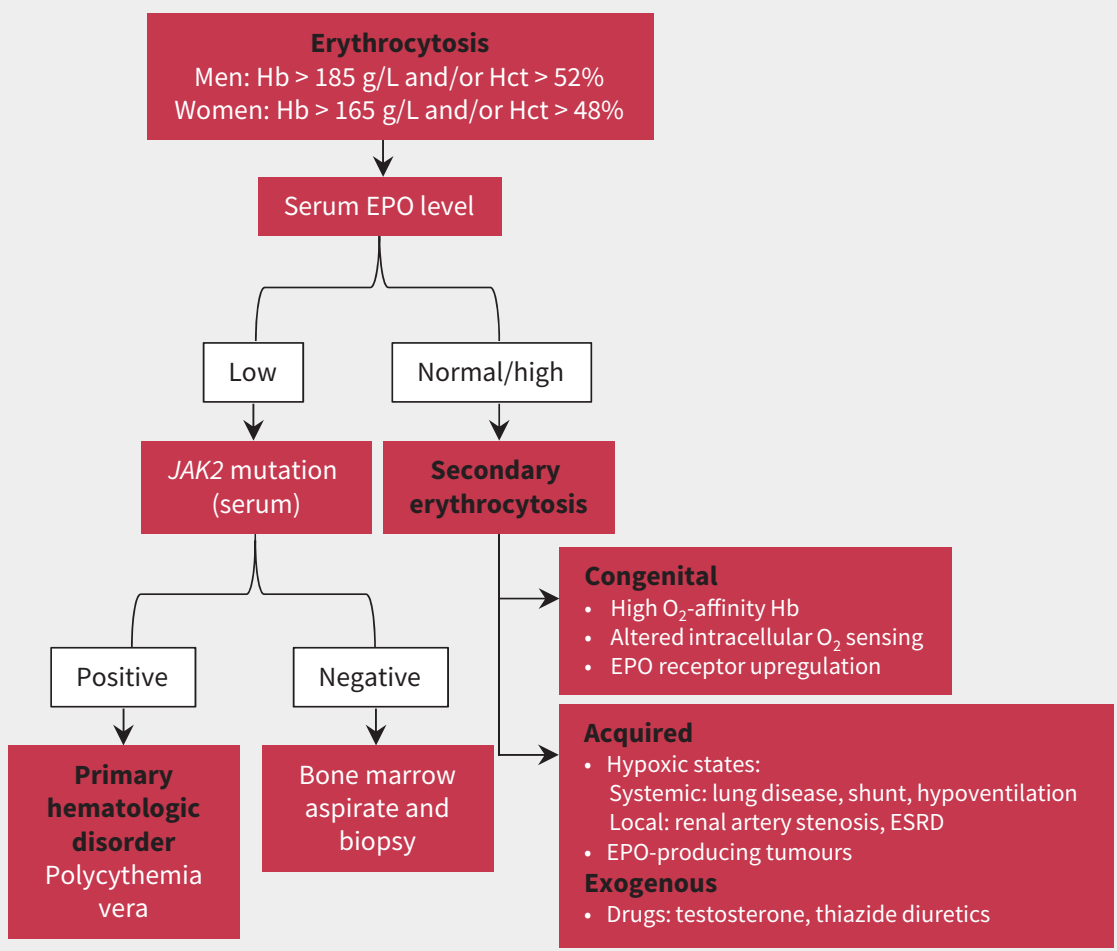

Figure 2: A generalized approach to erythrocytosis. ${ }^{4} \mathrm{EPO}=$ erythropoietin, $\mathrm{ESRD}=$ end-stage renal disease, $\mathrm{Hb}=$ hemoglobin, $\mathrm{Hct}=$ hematocrit. late erythropoietin, including darbopoietin, thiazide diuretics or androgen therapy (i.e., testosterone or anabolic steroids) are also common acquired causes for erythropoiesis. Alternatively, erythropoietin secretion can be pathologic in erythropoietin-producing malignant diseases such as renal cell carcinoma, hepatocellular carcinoma and pheochromocytoma. ${ }^{4}$

Erythrocytosis can result in symptoms of hyperviscosity, including chest and abdominal pain, weakness, fatigue, headache, blurred vision and paresthesias. Thrombosis is also an important consequence of erythrocytosis. A 34-year follow-up of the cohort of the Framingham study found an association between higher hematocrit and risk of cardiovascular mortality and morbidity. ${ }^{5}$ In addition to arterial thrombosis, an association between elevated hematocrit and venous thromboembolism has been recognized among patients with primary and secondary polycythemia, and even patients with an elevated baseline hematocrit who do not meet criteria for the classical definition of erythrocytosis. ${ }^{6}$ Although this relation has not been investigated in prospective randomized controlled trials, the US Food and Drug Administration has warned about the risk of venous thromboembolism, heart attack and stroke in patients using testosterone products. ${ }^{7}$

\section{Testosterone-induced erythrocytosis}

Interestingly, exogenous testosterone was used initially as a treatment for anemia. Recent understanding of hematopoiesis shows that estradiol, an aromatized form of testosterone, is responsible for an increase in hematopoietic stem cell proliferation and survival. In addition, testosterone increases erythropoiesis by increasing iron availability via reduced hepcidin levels, a hormone responsible for iron sequestration. ${ }^{8}$

An American study that evaluated the effects of graded doses of testosterone on erythropoiesis found that the percentage of hematocrit started to increase within one month of the 
start of treatment and continued to increase after three months in a linear dose-dependent manner. ${ }^{9}$ The study also reported that increases in hematocrit were exaggerated in older men (6075 years of age) versus young men (19-35 years of age). For example, $42 \%$ of men in the younger group who were taking a $125 \mathrm{mg}$ dose achieved peak hematocrit percentages after 12 weeks compared with $75 \%$ in the older group.

The American practice guideline on testosterone therapy recommends against the use of testosterone in patients with hematocrit above $50 \%$ or untreated obstructive sleep apnea, ${ }^{3}$ whereas the European guideline on male hypogonadism suggests that testosterone therapy is contraindicated at a hematocrit greater than $54 \% .{ }^{10}$ Although the most recent Canadian guideline does not advise on a hematocrit threshold above which testosterone therapy should be avoided, it recommends that hematocrit be measured at baseline, three to six months and annually, ${ }^{2}$ which is in keeping with the American guideline. ${ }^{3}$

In addition to monitoring of hematocrit percentage during testosterone replacement, physicians should reevaluate indications for testosterone to ensure that patients are receiving clinical benefit, warranting ongoing treatment. In addition, initiation of testosterone therapy should be limited to those patients who show both clinical and biochemical evidence of androgen deficiency to avoid complications caused by unnecessary drug exposure. ${ }^{2,3}$

Evidence-based guidelines on the management of erythrocytosis secondary to testosterone are lacking, and current guidelines based on expert consensus are variable. At a hematocrit percentage above $54 \%$, stopping testosterone is advised by the American guideline, ${ }^{3}$ whereas the European guideline suggests that phlebotomy should be considered at this level. ${ }^{10}$ This hematocrit value was derived from the Framingham cohort study. If the hematocrit percentage drops to less than $50 \%$, and no other secondary causes of erythrocytosis are found, testosterone can be restarted at lower doses. ${ }^{3}$

Although dose reduction or discontinuation may be appropriate for some patients, patients with symptoms of hyperviscosity may benefit from phlebotomy. For patients requiring ongoing testosterone treatment, transdermal formulations (with appropriate monitoring) may be a reasonable alternative to parenteral formulations, which are associated with higher hematocrit percentages. ${ }^{11}$

\section{References}

1. Barbui T, Thiele J, Vannucchi A, et al. Rationale for revision and proposed changes of the WHO diagnostic criteria for polycythemia vera, essential thrombocythemia and primary myelofibrosis. Blood Cancer J 2015;5:e337.

2. Morales A, Bebb RA, Manjoo P, et al. Diagnosis and management of testosterone deficiency syndrome in men: clinical practice guideline. CMAJ 2015;187:1369-77.
3. Bhasin S, Cunningham GR, Hayes FJ, et al. Testosterone therapy in men with androgen deficiency syndromes: an Endocrine Society clinical practice guideline. J Clin Endocrinol Metab 2010;95:2536-59.

4. Keohane C, McMullin MF, Harrison C. The diagnosis and management of erythrocytosis. BMJ 2013;347:f6667.

5. Gagnon DR, Zhang TJ, Brand FN, et al. Hematocrit and the risk of cardiovascular disease - the Framingham study: a 34-year follow-up. Am Heart J 1994;127:674-82.

6. Braekkan SK, Mathiesen EB, Njolstad I, et al. Hematocrit and risk of venous thromboembolism in a general population. The Tromso study. Haematologica 2010;95:270-5.

7. Testosterone products: FDA/CDER statement - risk of serious blood clots. Silver Spring (MD): US Food and Drug Administration; 2014. Available: http://fda.gov/ Safety/MedWatch/SafetyInformation/SafetyAlertsforHumanMedicalProducts/ ucm402054.htm (accessed 2017 Sept. 28).

8. Bachman E, Travison TG, Basaria S, et al. Testosterone induces erythrocytosis via increased erythropoietin and suppressed hepcidin: evidence for a new erythropoietin/hemoglobin set point. J Gerontol A Biol Sci Med Sci 2014;69:725-35.

9. Coviello AD, Kaplan B, Lakshman KM, et al. Effects of graded doses of testosterone on erythropoiesis in healthy young and older men. J Clin Endocrinol Metab 2008; 93:914-9.

10. Dohle GR, Arver S, Bettocchi C, et al. Guidelines on male hypogonadism. Arnhem (The Netherlands): European Association of Urology; 2012. Available: http://uroweb .org/wp-content/uploads/EAU-Guidelines-Male-Hypogonadism-2015.pdf (accessed 2017 July 29).

11. Siddique H, Smith JC, Corrall RJM. Reversal of polycythaemia induced by intramuscular androgen replacement using transdermal testosterone therapy. Clin Endocrinol (Oxf) 2004;60:143-5.

\section{Competing interests: None declared.}

This article has been peer reviewed.

The authors have obtained patient consent.

Affiliations: Department of Medicine, Divisions of Internal Medicine (Cervi) and Hematology (Balitsky), McMaster University, Hamilton, Ont.

Contributors: Andrea Cervi and Amaris Balitsky contributed equally to the conceptual design of the case summary and discussion topics. Both authors drafted, edited and critically revised the manuscript; gave final approval of the version to be published; and agreed to be accountable for all aspects of the work.

Acknowledgements: The authors thank Drs. Mark Crowther, Deborah Siegal and Wendy Lim for their helpful comments on earlier drafts of this manuscript.

Correspondence to: Andrea Cervi, andrea.cervi@medportal.ca

The section Cases presents brief case reports that convey clear, practical lessons. Preference is given to common presentations of important rare conditions, and important unusual presentations of common problems. Articles start with a case presentation (500 words maximum), and a discussion of the underlying condition follows ( 1000 words maximum). Visual elements (e.g., tables of the differential diagnosis, clinical features or diagnostic approach) are encouraged. Consent from patients for publication of their story is a necessity. See information for authors at www.cmaj.ca. 